PREPARED FOR THE U.S. DEPARTMENT OF ENERGY, UNDER CONTRACT DE-AC02-76CH03073

PPPL-3554

PPPL-3554

UC-70

Electron Acceleration in the Field-reversed Configuration (FRC)

by Slowly Rotating Odd-parity Magnetic Fields (RMF $)$

by

Alan H. Glasser and Samuel A. Cohen

April 2001

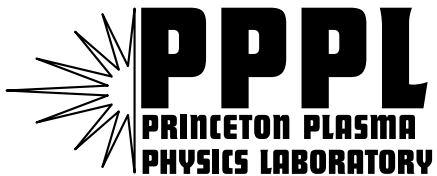

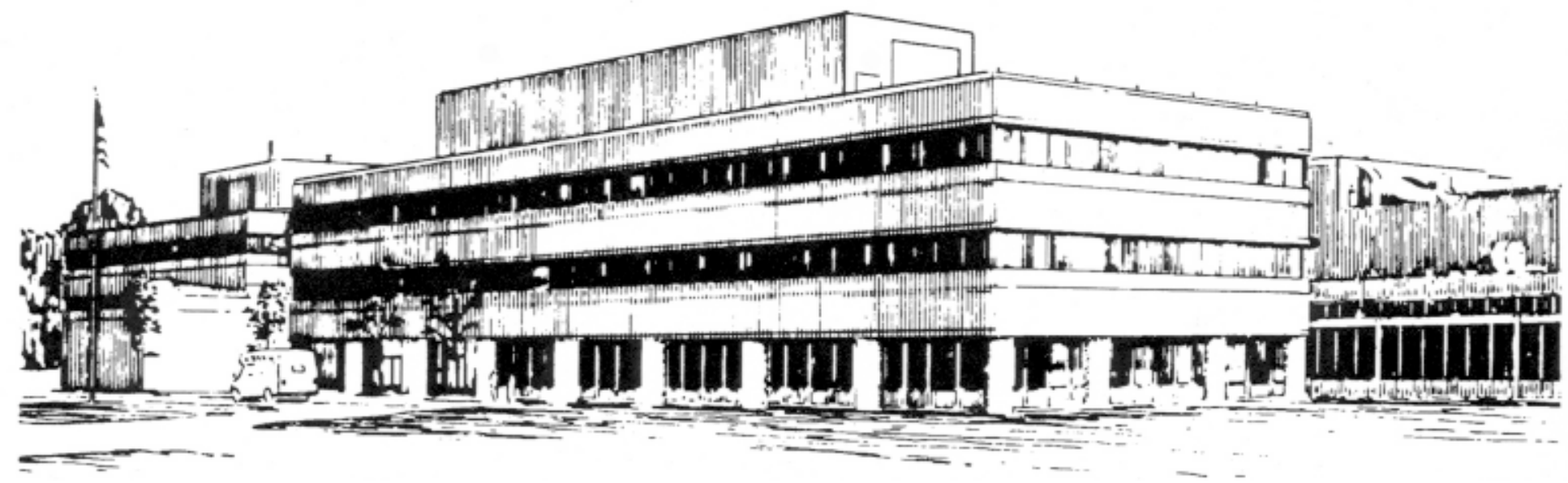

PRINCETON PLASMA PHYSICS LABORATORY PRINCETON UNIVERSITY, PRINCETON, NEW JERSEY 


\section{PPPL Reports Disclaimer}

This report was prepared as an account of work sponsored by an agency of the United States Government. Neither the United States Government nor any agency thereof, nor any of their employees, makes any warranty, express or implied, or assumes any legal liability or responsibility for the accuracy, completeness, or usefulness of any information, apparatus, product, or process disclosed, or represents that its use would not infringe privately owned rights. Reference herein to any specific commercial product, process, or service by trade name, trademark, manufacturer, or otherwise, does not necessarily constitute or imply its endorsement, recommendation, or favoring by the United States Government or any agency thereof. The views and opinions of authors expressed herein do not necessarily state or reflect those of the United States Government or any agency thereof.

\section{Availability}

This report is posted on the U.S. Department of Energy's Princeton Plasma Physics Laboratory Publications and Reports web site in Calendar Year 2001. The home page for PPPL Reports and Publications is: http://www.pppl.gov/pub_report/

DOE and DOE Contractors can obtain copies of this report from:

U.S. Department of Energy

Office of Scientific and Technical Information

DOE Technical Information Services (DTIS)

P.O. Box 62

Oak Ridge, TN 37831

Telephone: (865) 576-8401

Fax: (865) 576-5728

Email: reports@adonis.osti.gov

This report is available to the general public from:

National Technical Information Service

U.S. Department of Commerce

5285 Port Royal Road

Springfield, VA 22161

Telephone: 1-800-553-6847 or

(703) 605-6000

Fax: (703) 321-8547

Internet: http://www.ntis.gov/ordering.htm 


\title{
Electron acceleration in the field-reversed configuration (FRC) by slowly rotating odd-parity magnetic fields $\left(\mathrm{RMF}_{\mathrm{o}}\right)$
}

\author{
Alan H. Glasser, Los Alamos National Laboratory, Los Alamos, NM 87545 \\ Samuel A. Cohen, Princeton Plasma Physics Laboratory, Princeton, NJ 08543
}

\begin{abstract}
The trajectories of individual electrons are studied numerically in a 3D, prolate, FRC equilibrium magnetic geometry with added small-amplitude, slowly rotating, odd-parity magnetic fields $\left(\mathrm{RMF}_{\mathrm{o}} \mathrm{s}\right)$. $\mathrm{RMF}_{\mathrm{o}} \mathrm{s}$ cause electron heating by toroidal acceleration near the O-point line and by field-parallel acceleration away from it, both followed by scattering from magnetic-field inhomogeneities. Electrons accelerated along the O-point line move antiparallel to the FRC's current and attain average toroidal angular speeds near that of the $\mathrm{RMF}_{\mathrm{o}}$, independent of the sense of $\mathrm{RMF}_{\mathrm{o}}$ rotation. A conserved transformed Hamiltonian, dependent on electron energy and $\mathrm{RMF}_{\mathrm{o}}$ sense, controls electron flux-surface coordinate.
\end{abstract}

A recent publication ${ }^{1}$ considered the orbits of individual ions in an FRC with smallamplitude odd-parity ${ }^{2}$ rotating magnetic fields $\left(\mathrm{RMF}_{\mathrm{o}} \mathrm{s}\right)$ in the Ion Cyclotron Range of Frequencies (ICRF). That paper showed that ions can be accelerated to thermonuclear energies in a modest-size device without loss of confinement. In this paper we study the motion of electrons in the same system, with the RMF o still in the ICRF, far below the electron cyclotron frequency range. Because of the mass difference, the physical mechanisms responsible for electron heating are far different from those described for the ions whose heating can be viewed as a hybrid of cyclotron resonance and Fermi acceleration. In contrast, the physical picture of electron heating combines acceleration by slowly-varying electric fields with scattering from field inhomogeneities. We show that this essential difference results in novel phenomena. All other conditions noted in Ref.1 also apply here.

These results are important for fusion reactor design. The FRC, recognized to have many attractive technical and scientific features as a power plant, ${ }^{3}$ lacks proven methods to heat electrons and drive sustained currents, particularly on the magnetic axis. ${ }^{4-7}$ This paper shows the physics by which $\mathrm{RMF}_{\mathrm{o}} \mathrm{s}$ in the ICRF can heat electrons and drive an on-axis electron current.

A central feature of the electron-heating mechanism is collisionless scattering from the non-uniform magnetic field structure, first described by Speiser in 2-D. ${ }^{8}$ In an FRC with elongation $\kappa \equiv z_{s} / r_{s},\left(r_{s}=\right.$ separatrix radius and $\pm z_{s}=$ axial positions of the $\mathrm{X}$ points $)$, the magnetic field strength $|B|$ drops by a factor $\geq 2 \kappa$ in going from the mid-plane to the extrema of a flux surface. As the electron approaches the sharp curve at an extremum, its gyro-radius, $\rho_{L}$, increases and the radius of field curvature, $r_{c}$ decreases. If $\rho_{L} / r_{c}>$ 0.01 , the adiabatic invariance of magnetic moment is violated where the field reverses direction; the energy is equipartitioned between parallel and perpendicular motions. In this scattering, no shift in the electron's flux-surface coordinate occurs. Our simulations, performed in 3-D, show new phenomena.

In the computer code, $\mathrm{RMF}_{1.13}$, we use an adaptive integrator ${ }^{9}$ to integrate the six coupled nonlinear ordinary differential equations comprising Hamilton's equations, ${ }^{10}$ 


$$
\dot{q}_{i}=\partial H / \partial p_{i}, \quad \dot{p}_{i}=-\partial H / \partial q_{i}, \quad i=r, z, \phi
$$

with $H$ the Hamiltonian and $q_{i}$ and $p_{i}$ the canonical coordinates and momenta. Vector potentials for the Solov'ev $\mathrm{FRC}^{11}$ and the $\mathrm{RMF}_{\mathrm{o}} \mathrm{s}^{1}$ are respectively given by

$$
\begin{gathered}
r A_{\phi, \mathrm{FRC}} \equiv \Psi_{\mathrm{FRC}}=\Psi_{0}\left(r^{2} / r_{s}^{2}\right)\left(1-r^{2} / r_{s}^{2}-z^{2} / z_{s}^{2}\right), \\
\left\{A_{r}, A_{z}, A_{\phi}\right\}_{\text {odd }}=\left(2 B_{R} / k\right)\left\{I_{0}(\xi) \cos k z \sin \psi,-I_{1}(\xi) \sin k z \sin \psi, I_{0}(\xi) \cos k z \cos \psi\right\}
\end{gathered}
$$

with $\Psi_{0}=B_{a} r_{s}^{2} / 2, B_{a}$ the FRC field at $z=r=0, B_{R}$ the RMF field amplitude, $k=$ $l \pi / \kappa r_{s}$ the $\mathrm{RMF}_{\mathrm{o}}$ wave number, $l$ the $\mathrm{RMF}_{\mathrm{o}}$ axial mode number, $\xi \equiv k r, m_{e}$ the electron mass, $q_{e}=-e$ the electron charge, $\psi \equiv \phi-\omega_{R} t, \omega_{c e} \equiv q_{e} B_{a} / m_{e} c$ the electron cyclotron frequency, $\omega_{R}$ the $\mathrm{RMF}_{\mathrm{o}}$ frequency, and $I_{m}$ modified Bessel functions. An electrostatic potential $\varphi\left(\Psi_{\mathrm{FRC}}\right)$ may be included in $H$, as suggested by fluid models. ${ }^{12}$ We have studied $\varphi$ values up to $10 \mathrm{keV}$ and seen that the primary results are unaltered. For simplicity, all results reported here had $\varphi=0$.

Because $H$ depends on $\phi$ and $t$ only through $\psi$, it follows that the transformed Hamiltonian

$$
K \equiv H-\omega_{R} p_{\phi}
$$

is conserved. ${ }^{10} \mathrm{~K}$ is used to monitor the accuracy of numerical integration and, more importantly, is shown below to control electron motion across flux surfaces.

In a typical $\mathrm{RMF}_{1.13}$ run, a 100-eV electron is initialized at a position inside the FRC's separatrix. Other initial parameters are the angles of the electron's velocity, and the mode structure, phase, frequency, and amplitude of the $\mathrm{RMF}_{\mathrm{o}}$. Electrons with $100 \mathrm{eV}$ perform cyclotron orbits, unless they are very close to the O-point null line, in which case they may perform null-line-crossing betatron orbits. ${ }^{13,14}$ In an FRC, electron cyclotron orbits drift in one toroidal direction, parallel to the FRC's current, thus reducing it, while betatron orbits move in the opposite direction, adding to the current. The sign of $\omega_{R}$ is positive when the $\mathrm{RMF}_{\mathrm{o}}$ rotates in the direction of the electron betatron motion.

For the reference FRC (RFRC) of Ref. 1, with $B_{a}=2 \times 10^{4} \mathrm{G}, r_{s}=10 \mathrm{~cm}$, and $\kappa=5$, Fig. 1 shows results of an $\mathrm{RMF}_{1.13}$ simulation for an electron initiated on a flux surface near the O-point line. The $\mathrm{RMF}_{\mathrm{o}}$ parameters were $\omega_{R} / \omega_{c e}=2 \times 10^{-4}$ and $B_{R}=20$ G. This sense of $R M F_{o}$ rotation, positive $\omega_{R} / \omega_{c e}$, is the same as known to drive current for even-parity $\mathrm{RMF}\left(\mathrm{RMF}_{e}\right)$. The electron energy is shown as a function of $\tau=t / \tau_{c e}$, time measured in units of the electron cyclotron period $\tau_{c e}=2 \pi / \omega_{c e}$ at $B_{a}$. The energy periodically spikes to above $4 \mathrm{keV}$ and then nearly returns to its pre-spike baseline value. A slow, secular increase in the baseline energy also occurs, reaching a maximum of about 2 $\mathrm{keV}$ at $\tau \approx 4 \times 10^{4}$. As the baseline energy rises, the heights of the spikes above the baseline decrease; the maximum energy attained (spikes plus baseline) also decreases. Extending the simulation to $\tau=10^{7}$ resulted in less than a $3 \%$ further increase in maximum energy attained. In this extended time period, the secular behavior of the baseline energy is not 
monotonic; its value varies between zero and about $3 \mathrm{keV}$. Numerical integration of the energy gain $\Delta \mathcal{E} \equiv \int_{0}^{t} q_{e} \mathbf{E} \cdot \mathbf{v}_{\mathbf{e}} d t$ shows that both the energy spikes and the secular energy gain come predominantly from the azimuthal (toroidal) component $E_{\phi}$.

Figure 2 shows, with finer time resolution, the same electron's energy, axial position $z$, radial position $r$, and azimuthal position, $\phi$. The spikes occur during betatron-type orbits, when the electron is near the $\mathrm{O}$-point position, $r=7.07 \mathrm{~cm}, z=0 \mathrm{~cm}$. The results of Speiser collisions can be seen, Fig. 2b, in the erratic motion near the $z$ extrema of the original flux surface, $\sim \pm 4 \mathrm{~cm}$. For example, numerous Speiser collisions occur during the interval $3700<\tau<5500$, causing the electron to linger near the positive $z$ extremum. The $\phi$ position, Fig. 2d, becomes increasingly more negative with $\tau$, dominated by steps due to the energetic betatron orbits. The time-averaged angular velocity $\langle\dot{\phi}\rangle$ has the same rotation sense as the $\mathrm{RMF}_{\mathrm{o}}$ and nearly (0.98) the same magnitude. This timeaveraged synchronous motion continues for very long times, beyond $\tau=10^{7}$. Electrons accelerated with a positive $\omega_{R} / \omega_{c e} \mathrm{RMF}_{\mathrm{o}}$ are well confined; they move towards the $\mathrm{O}$ point with increasing energy. Electrons initiated away from the $\mathrm{O}$ point are accelerated to high energy by the $E_{z}$ field; Speiser collisions cause isotropization of the energy. These electrons move towards the $\mathrm{O}$ point where the $E_{\phi}$ field can cause toroidal acceleration. As we show later, electrons initiated further from the $\mathrm{O}$ point eventually attain higher energy than those initiated nearer the $\mathrm{O}$ point.

Figure 3 shows $\mathrm{RMF}_{1.13}$ results for reversed rotation, $\omega_{R} / \omega_{c e}=-2 \times 10^{-4}$. Energy spikes of $\sim$ half the amplitude as for positive $\omega_{R} / \omega_{c e}$ occur during the early phase, $\tau<$ $3 \times 10^{4}$. Surprisingly, for this reversed $\mathrm{RMF}_{\mathrm{o}}$ rotation direction, the electron initially moves with nearly the same average toroidal angular speed (Fig. 3b) as the $\mathrm{RMF}_{\mathrm{o}}$, but with the opposite rotational sense, contrary to the commonly invoked physical picture of electrons frozen to the rotating magnetic field. ${ }^{14-16}$ At times when the energy is above $\sim 5 \mathrm{keV}$, the spikes are absent.

For negative $\omega_{R} / \omega_{c e}$, electrons accelerated to higher energies move further away from the $\mathrm{O}$ point. The further an electron moves from the $\mathrm{O}$ point, the larger the fraction of its total kinetic energy, $\mathcal{E}_{t}$, comes from $E_{z}$. At larger energies and smaller values of $\Psi_{\text {FRC }}$, the average toroidal angular speed of an electron is a very small fraction of $\omega_{R}$, Fig. $3 \mathrm{~b}$. Because of this outward motion with increasing energy, the energy of those electrons initiated near the $\mathrm{O}$ point eventually increases to much above that obtained for the same initial position with $\omega_{R} / \omega_{c e}=+2 \times 10^{-4}$, to about $10 \mathrm{keV}$ at $\tau=10^{5}$ and $40 \mathrm{keV}$ at $\tau=10^{6}$.

That electrons heated at negative $\omega_{R} / \omega_{c e}$ move away from the $\mathrm{O}$ point while those heated at positive $\omega_{R} / \omega_{c e}$ move towards the $\mathrm{O}$ point can be understood from the constancy of $K$, Eq. (4). The dominant part of $p_{\phi}=m r^{2} \dot{\phi}+q_{e} r A_{\phi} / c$ is $q_{e} \Psi_{\mathrm{FRC}} / c$. A change of the sign of $\omega_{R} / \omega_{c e}$ requires a corresponding change of sign of the time derivative of $\Psi_{\mathrm{FRC}}$ to conserve $K$ with changing $H$.

The energy spikes can be understood in terms of the combined effects of the FRC's static magnetic field and the time-dependent $\mathrm{RMF}_{\mathrm{o}}$-generated electric field. Because the FRC has a minimum-B geometry with $B=0$ at the $\mathrm{O}$ point, $r=r_{o}$ and $z=0$, an electron there is guided toroidally around the FRC in a near-circular orbit of radius $r=r_{o}$, 
with small axial and radial betatron modulations. ${ }^{13,14}$ The $\mathrm{RMF}_{\text {o }}$ produces an azimuthal electric field via the time derivative $-\left(\partial A_{\phi, o d d} / \partial t\right) / c$ of Eqn. (3), causing the electron to accelerate azimuthally. The $\cos \psi$ term in $A_{\phi, o d d}$, Eq. (3), causes the electric field to reverse sign twice as $\phi$ increases by $2 \pi$. A sufficiently fast electron will proceed in this field to where the $E_{\phi}$ field reverses direction, i.e., about half way around the FRC. At that point, deceleration will begin and proceed until nearly all the energy gained is lost. If a non-adiabatic scattering event occurs before all the energy is lost, heating occurs. Energy spikes occur for both directions of $\mathrm{RMF}_{\mathrm{o}}$ rotation because both produce an $E_{\phi}$ with the same toroidal behavior. Energy spikes are larger for positive $\omega_{R} / \omega_{c e}$ because the $R M F_{o}$ rotates in the direction of the betatron orbits so electrons stay longer in phase with the $E_{\phi}$ than for negative $\omega_{R} / \omega_{c e}$.

From a series of over $2000 \mathrm{RMF}_{1.13}$ runs, we determine how the maximum energy $\mathcal{E}_{\text {max }}$ attained by an electron depends on its initial position $r_{\text {init }}$, the strength and frequency of the $\mathrm{RMF}_{\mathrm{o}}$, the rotation sense of the $\mathrm{RMF}_{\mathrm{o}}$, and the duration of the $\mathrm{RMF}_{1.13}$ run. For $\omega_{R} / \omega_{c e}=2 \times 10^{-4}$, Fig. 4a shows, for four strengths of $\mathrm{RMF}_{\mathrm{o}}$, the maximum energy attained in time $\tau=5 \times 10^{4}$ as a function of $r_{\text {init }}$, normalized to the O-point radius, $\rho_{\text {init }} \equiv r_{\text {init }} / r_{o}$. At $B_{R}=1 \mathrm{G}$, a peak in $\mathcal{E}_{\max }$ appears at $\rho_{\text {init }}=1$, exceeding the off-Opoint heating by about a factor of 5 . The height of $\mathcal{E}_{\max }$ increases and the radial width, $\delta_{p}$, of the near-O-point heating broadens with increasing $B_{R}, \omega_{R}$, and $\tau,\left(\delta_{p} \propto \tau^{0.2}\right)$, but the central peak collapses. The smooth sunken peak that develops is a robust feature of $\mathrm{RMF}_{1.13}$ simulations for positive $\omega_{R} / \omega_{c e}$. It can be understood from Eq. 2 and 4 . When $\omega_{R} / \omega_{c e}$ is negative, a sunken peak in $\mathcal{E}_{\max }$ does not occur because electrons initiated near the $\mathrm{O}$ point move to lower $\Psi_{\mathrm{FRC}}$ and are not resricted in energy by Eq. 4.; $\mathcal{E}_{\max }$ is then roughly independent of $\rho_{\text {init }}$ and equal to the value obtained with positive $\omega_{R} / \omega_{c e}$ for electrons initiated at the separatrix radius.

The ratio $\alpha^{*} \equiv<\dot{\phi}>/ \omega_{R}$ is shown in Fig. $4 \mathrm{~b}$ for positive $\omega_{R} / \omega_{c e}$ as functions of $B_{R}$ and $\rho_{\text {init }}$. (Positive $\alpha^{*}$ means electron azimuthal angular velocity in the same direction as the $\mathrm{RMF}_{\mathrm{o}}$ rotation.) Synchronous rotation occurs in the same regions as the sunken peaks in Fig. 4a. The plateaus in $\alpha^{*}$ mark the initial positions of the electrons' trajectories, which is not the same as the regions where motion synchronous with the $\mathrm{RMF}_{\mathrm{o}}$ occurs. Electrons move synchronously with the $\mathrm{RMF}_{\mathrm{o}}$ only close to the $\mathrm{O}$ point, typically within $\pm 5 \% r_{o}$ and $\pm 10 \% z_{s}$.

Speiser scattering might be invoked to estimate a non-Ohmic resistivity, for example, by assuming that Speiser collisions occur each time an electron moves a distance $\sim \kappa r_{s}$ along the magnetic field. This simple model predicts that the ratio of Speiser collision frequency, $\nu_{s}$, to the Spitzer collision frequency, $\nu_{z}$, is $\nu_{s} / \nu_{z} \sim 10^{12} \mathcal{E}_{e}^{2} / n_{e} r_{s} \kappa$. This could provide an explantion for the enhanced resistivity seen in both $\mathrm{FRC}^{4}$ and spheromak ${ }^{17}$ experiments. However, the results shown in Fig. 2 indicate that this model oversimplifies the resistivity, most clearly for RMF-heated FRCs, because the energy gain saturates for positive $\omega_{R} / \omega_{c e}$. For negative $\omega_{R} / \omega_{c e}, \mathcal{E}_{t}$ grows slowly, $\propto \tau^{0.5}$.

In summary, we have shown with a full 3-D model that, if $\mathrm{RMF}_{\mathrm{o}}$ penetration is full, small-amplitude odd-parity rotating magnetic fields can be used in modest-sized FRC devices to heat electrons to fusion-relevant energies. For the standard $\mathrm{RMF}_{\mathrm{o}}$ rotation 
sense, electrons intiated away from the $\mathrm{O}$ point are accelerated by the $E_{z}$ field and their energy is redistributed amongst the degrees- of-freedom by Speiser collisions. The orbits of these electrons move towards the $\mathrm{O}$ point as their energy increases. Near the $\mathrm{O}$ point they are accelerated predominantly by the $E_{\phi}$ field, to synchronous motion with the $\mathrm{RMF}_{\mathrm{o}}$. For the opposite sense of $\mathrm{RMF}_{\mathrm{o}}$ rotation, electrons near the magnetic axis are also accelerated to angular speeds equal to that of the $\mathrm{RMF}_{\mathrm{o}}$, but their sense of rotation is opposite. As these electrons heat, they move away from the $\mathrm{O}$ point and their toroidal drift speed decreases. The role of the conserved transformed Hamiltonian, K, in determining electron flux-surface coordinate is shown. These results are relevant to a compact magnetic-fusionreactor design.

We thank T. Carter, T.K. Chu, J. Finn, J. Foley, T. Kornack and T. Munsat for useful discussions. This work was supported in part by U.S. Department of Energy Contract No. DE-AC02-76-CHO-3073. 


\section{References}

1. S.A. Cohen and A.H. Glasser, Phys. Rev. Lett. 85, 5114 (2000).

2. S.A. Cohen and R.D. Milroy, Phys. Plasmas 7, 2539 (2000).

3. D.C.Barnes, M. Binderbauer, R.D. Brooks, et al., Fusion Technology 30, 116 (1996).

4. J.T. Slough and K. E. Miller, Phys. Plasmas 7, 1945 (2000).

5. R.D. Milroy, Phys. Plasmas 7, 4135 (2000).

6. A.L. Hoffman, Nucl. Fusion 40, 1523 (2000).

7. A. Hassam, R. Kulsrud, R.J. Goldston et al., Phys. Rev. Lett. 83, 2969 (2000).

8. T.W. Speiser, J. Geophys. Res. 70, 4219 (1965).

9. A. C. Hindmarsh, "ODEPACK, A Systematized Collection of ODE Solvers", in Scientific Computing, R. S. Stepleman et al., Editors (North-Holland, Amsterdam, 1983), pp. 55-64.

10. H. Goldstein, Classical Mechanics, 2nd ed., (Addison-Wesley, Reading, MA, 1980).

11. L.S. Solov'ev, Rev. Plasma Phys. 6, 239 (1976).

12. W.N. Hugrass, J. Plasma Phys. 28, 369 (1982).

13. M.Y. Yang and G.H. Miley, Nucl. Fusion. 79, 39 (1979).

14. W.N. Hugrass and I. R. Jones, J. Plasma Physics 29, 155 (1983).

15. H.A. Blevin and P.C. Thonemann, Nucl. Fusion: 1962 Supplement, Part 1, 55.

16. A.L. Hoffman, Phys. Plasmas 5, 979 (1998).

17. M. Yamada, H. Ji, S. Hsu, et al., Phys. Rev. Lett. 78, 3117 (1997).

\section{Figure captions}

1. Calculated electron energy as a function of scaled dimensionless time, $\tau=\omega_{c e} t / 2 \pi$ in an FRC with $B_{a}=2 \times 10^{4}, r_{s}=10 \mathrm{~cm}$ and $\kappa=5$, for odd-parity RMFs with $\omega_{R} / \omega_{c e}=2 \times 10^{-4}$ and $B_{R}=20 \mathrm{G}$.

2. Calculated electron a) energy, b) axial $z$, c) radial $r$, and d) azimuthal $\phi$ positions, on an expanded $\tau$ scale from Fig.1. The energy spikes are coincident with orbits near the O-point line and large negative-going steps in $\phi$.

3. a) Electron energy vs. $\tau$ in the FRC for $\mathrm{RMF}_{\mathrm{o}}$ with $\omega_{R} / \omega_{c e}=-2 \times 10^{-4}$ and $B_{R}=20$ G. Spikes are seen at at low energy, $<4 \mathrm{keV}$. b) Azimuthal position vs. $\tau$. When spikes occur, the azimuthal speed is close to that of the $\mathrm{RMF}_{\mathrm{o}}$, but the diirections of motion are opposite.

4. a) Maximum electron energy attained, $\mathcal{E}_{\max }$, vs. initial normalized electron radial position $\rho_{\text {init }}$, at $\omega_{R} / \omega_{c e}=2 \times 10^{-4}$ for 4 values of $B_{R}: 1,4,16$, and $64 \mathrm{G}$. The duration of each $\mathrm{RMF}_{1.13}$ run was $\tau=3 \times 10^{4}$. b) $\alpha^{*}$, ratio of time-averaged electron azimuthal angular velocity to $\omega_{R}$, versus $\rho_{\text {init }}$. 


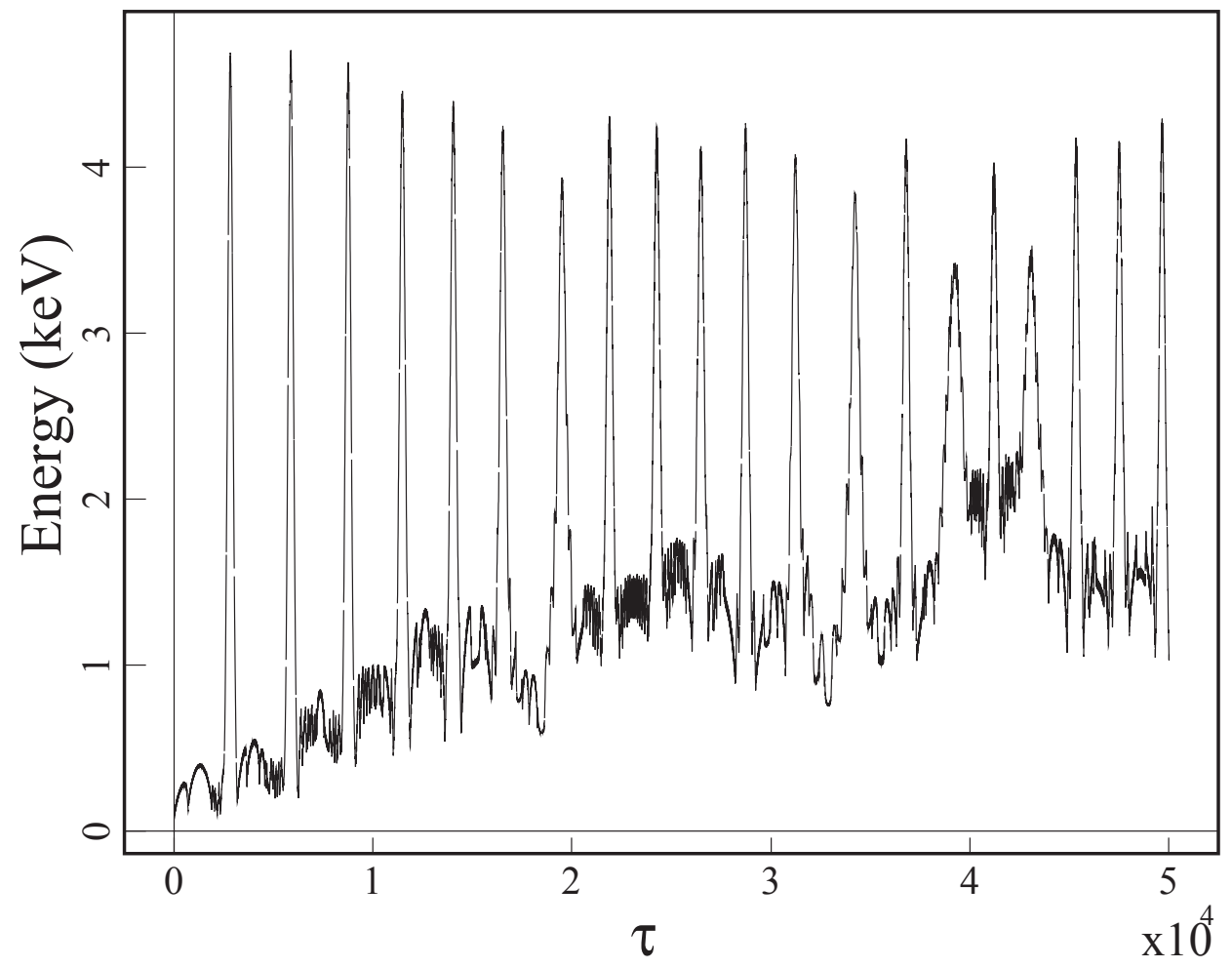




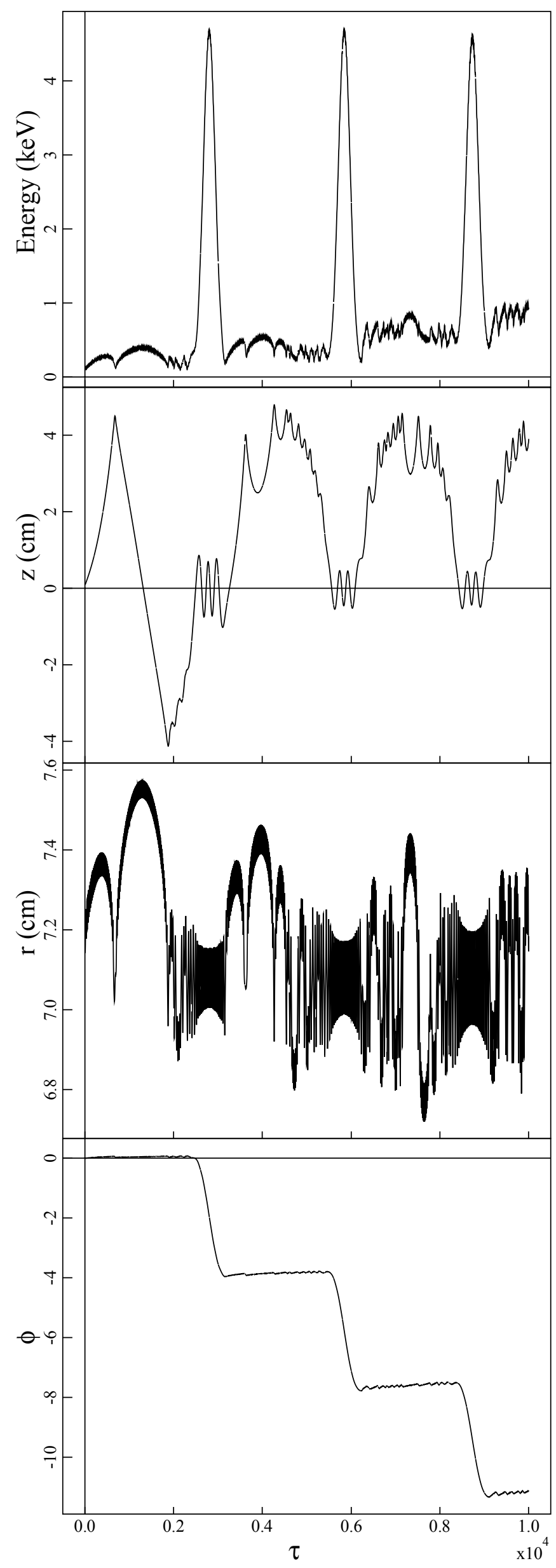




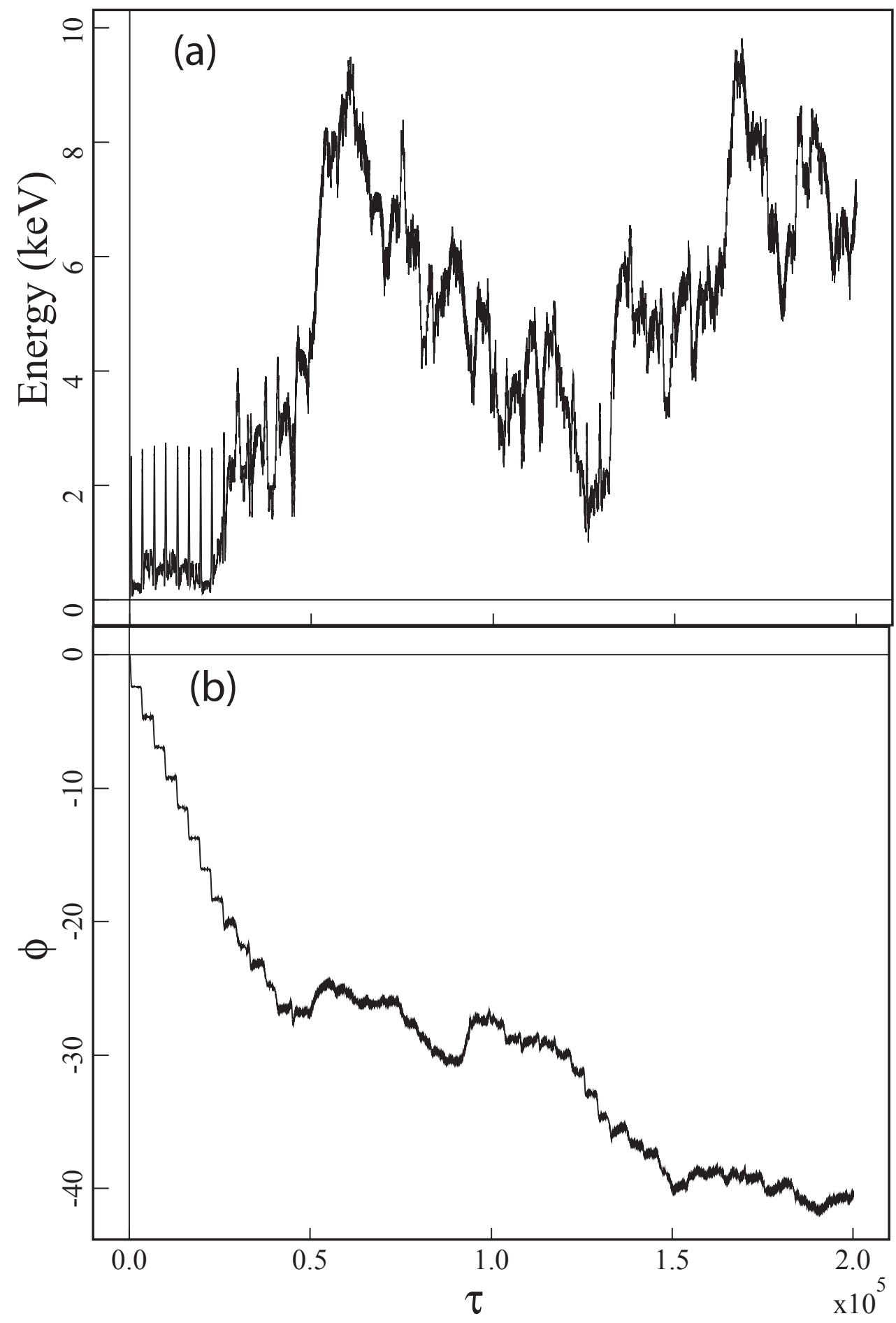




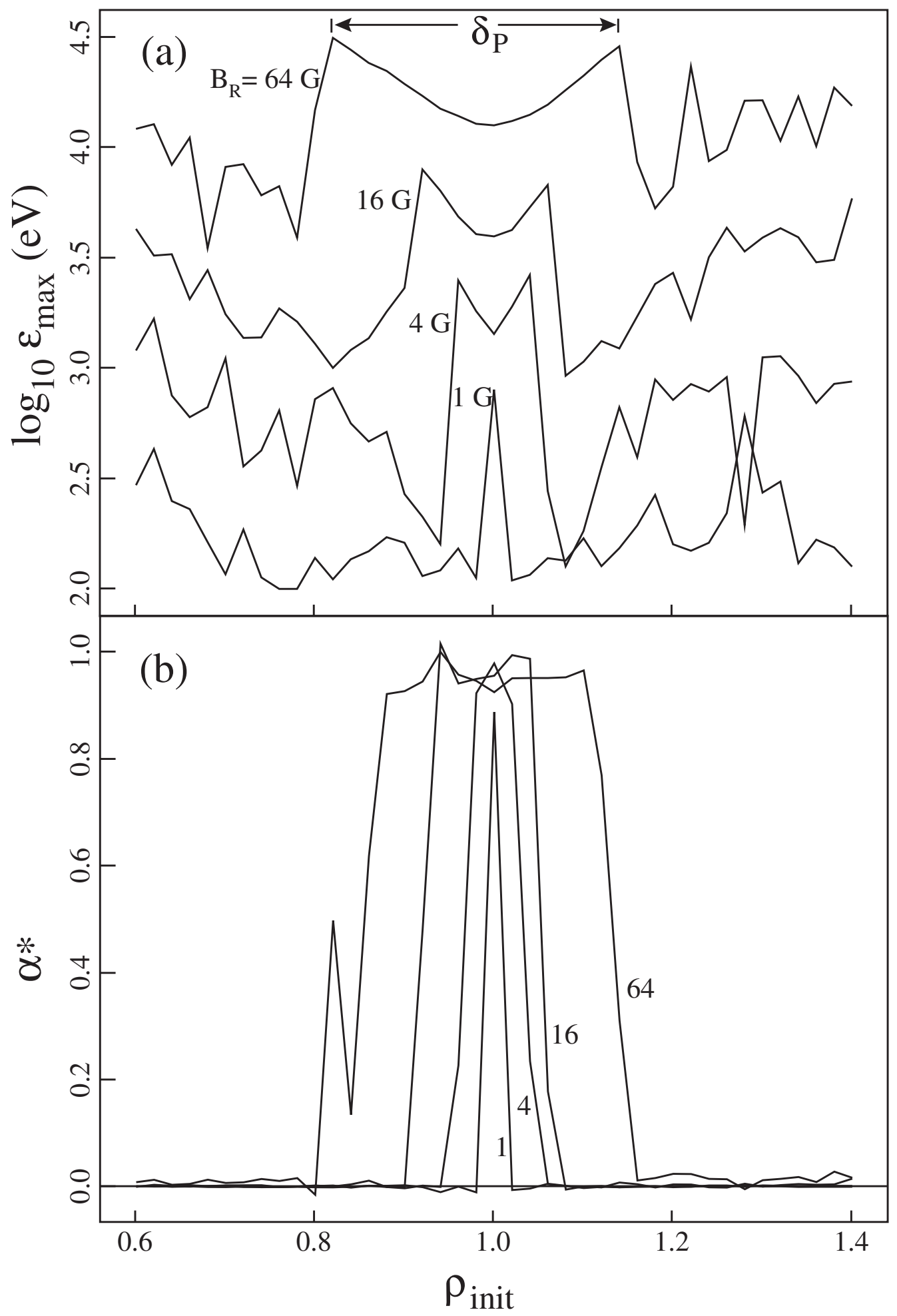




\section{External Distribution}

Plasma Research Laboratory, Australian National University, Australia

Professor I.R. J ones, Flinders University, Australia

Professor J oão Canalle, Instituto de Fisica DEQ/IF - UERJ , Brazil

Mr. Gerson O. Ludwig, Instituto Nacional de Pesquisas, Brazil

Dr. P.H. Sakanaka, Instituto Fisica, Brazil

The Librarian, Culham Laboratory, England

Library, R61, Rutherford Appleton Laboratory, England

Mrs. S.A. Hutchinson, JET Library, England

Professor M.N. Bussac, Ecole Polytechnique, France

Librarian, Max-Planck-Institut für Plasmaphysik, Germany

J olan Moldvai, Reports Library, MTA KFKI-ATKI, Hungary

Dr. P. Kaw, Institute for Plasma Research, India

Ms. P.J . Pathak, Librarian, Insitute for Plasma Research, India

Ms. Clelia De Palo, Associazione EURATOM-ENEA, I taly

Dr. G. Grosso, Instituto di Fisica del Plasma, Italy

Librarian, Naka Fusion Research Establishment, J AERI, J apan

Library, Plasma Physics Laboratory, Kyoto University, J apan

Research Information Center, National Institute for Fusion Science, J apan

Dr. O. Mitarai, Kyushu Tokai University, J apan

Library, Academia Sinica, Institute of Plasma Physics, People's Republic of China

Shih-Tung Tsai, Institute of Physics, Chinese Academy of Sciences, People's Republic of China

Dr. S. Mirnov, TRINITI, Troitsk, Russian Federation, Russia

Dr. V.S. Strelkov, Kurchatov Institute, Russian Federation, Russia

Professor Peter Lukac, Katedra Fyziky Plazmy MFF UK, Mlynska dolina F-2, Komenskeho Univerzita, SK-842 15 Bratislava, Slovakia

Dr. G.S. Lee, Korea Basic Science Institute, South Korea

Mr. Dennis Bruggink, Fusion Library, University of Wisconsin, USA

Institute for Plasma Research, University of Maryland, USA

Librarian, Fusion Energy Division, Oak Ridge National Laboratory, USA

Librarian, Institute of Fusion Studies, University of Texas, USA

Librarian, Magnetic Fusion Program, Lawrence Livermore National Laboratory, USA

Library, General Atomics, USA

Plasma Physics Group, Fusion Energy Research Program, University of California at San Diego, USA

Plasma Physics Library, Columbia University, USA

Alkesh Punjabi, Center for Fusion Research and Training, Hampton University, USA

Dr. W.M. Stacey, Fusion Research Center, Georgia Institute of Technology, USA

Dr. J ohn Willis, U.S. Department of Energy, Office of Fusion Energy Sciences, USA

Mr. Paul H. Wright, Indianapolis, Indiana, USA 
The Princeton Plasma Physics Laboratory is operated by Princeton University under contract with the U.S. Department of Energy.

\author{
Information Services \\ Princeton Plasma Physics Laboratory \\ P.O. Box 451 \\ Princeton, NJ 08543
}

Phone: 609-243-2750

Fax: 609-243-2751

e-mail: pppl_info@pppl.gov

Internet Address: http://www.pppl.gov 